Service social

\title{
Suicide des jeunes et homophobie en France : présentation d'une enquête et d'actions de prévention
}

\section{Jean-Michel Pugniere}

Volume 59, numéro 1, 2013

L'homophobie et les tentatives de suicide et la résilience chez les jeunes LGBTQ

URI : https://id.erudit.org/iderudit/1017477ar

DOI : https://doi.org/10.7202/1017477ar

Aller au sommaire du numéro

\section{Éditeur(s)}

École de service social de l’Université Laval

ISSN

1708-1734 (numérique)

Découvrir la revue

Citer cet article

Pugniere, J.-M. (2013). Suicide des jeunes et homophobie en France : présentation d'une enquête et d'actions de prévention. Service social, 59(1), 17-34. https://doi.org/10.7202/1017477ar
Résumé de l'article

De nombreuses recherches ont mis en évidence un lien significatif entre orientation sexuelle et suicide des adolescent-e-s / jeunes adultes (Beck et al., 2010). L'homophobie à laquelle les jeunes homo/bisexuel-le-s sont exposé-e-s est considérée comme l'hypothèse la plus sérieuse pour expliquer ce lien, mais, jusqu'ici, cette hypothèse n'avait jamais fait l'objet d'une recherche quantitative en France. Nous l'avons prise en compte dans le cadre d'une enquête réalisée sur la base d'un questionnaire informatisé auto-administré auquel ont répondu 475 filles et 426 garçons, âgé-e-s de 18 à 24 ans. Les résultats confirment la sursuicidalité des jeunes homo/bisexuelle-le-s et mettent en évidence l'impact de l'intimidation homophobe en milieu scolaire chez les adolescents et jeunes adultes de sexe masculin. Ce constat nous amène à évoquer le travail de prévention mené par les associations, notamment l'aide aux familles et les interventions en milieu scolaire proposées par l'association Contact. 


\title{
Suicide des jeunes et homophobie en France : présentation d'une enquête et d'actions de prévention
}

\author{
Jean-Michel Pugniere
}

\section{RÉSUMÉ}

De nombreuses recherches ont mis en évidence un lien significatif entre orientation sexuelle et suicide des adolescent-e-s / jeunes adultes (Beck et al., 2010). L'homophobie à laquelle les jeunes homo/bisexuel-le-s sont exposé-e-s est considérée comme l'hypothèse la plus sérieuse pour expliquer ce lien, mais, jusqu'ici, cette hypothèse n'avait jamais fait l'objet d'une recherche quantitative en France. Nous l'avons prise en compte dans le cadre d'une enquête réalisée sur la base d'un questionnaire informatisé auto-administré auquel ont répondu 475 filles et 426 garçons, âgé-e-s de 18 à 24 ans. Les résultats confirment la sursuicidalité des jeunes homo/bisexuelle-le-s et mettent en évidence l'impact de l'intimidation homophobe en milieu scolaire chez les adolescents et jeunes adultes de sexe masculin. Ce constat nous amène à évoquer le travail de prévention mené par les associations, notamment l'aide aux familles et les interventions en milieu scolaire proposées par l'association Contact.

Mots clés : Orientation sexuelle; suicide; adolescents; jeunes; homophobie; intimidation (bullying) en milieu scolaire; prévention; associations.

\begin{abstract}
Several studies put into light a significant link between sexual orientation and suicidal behaviors among teenagers and/or young adults. The homophobia to which young homo/bisexuals are exposed is considered the most serious hypothesis to explain this link. However quantitative studies have never been conducted on this hypothesis in France. We took it into account while carrying out a survey based on a self-administered questionnaire. Said survey was answered by 475 young women, and 426 young men aged 18-24.

Results confirmed oversuicidality among young homo/bisexuals, and highlight the extent of homophobic bullying in school, and its impact on male teenagers and young adults. This leads us to discuss the prevention work carried out by community groups, including assistance to families and school interventions proposed by the association Contact.
\end{abstract}

Key words: Sexual orientation; suicide; teenagers; young adults; homophobia; intimidation; school bullying; prevention.

En France, les conséquences psychologiques et sociales de l'homophobie constituent un domaine de recherche jusqu'ici peu étudié. Ces dernières années, les associations LGBT (Lesbiennes, Gays, Bisexuel-le-s et Transexuel-le-s), ainsi que le rapport des responsables de la ligne Azur (ligne d'écoute nationale au sujet de l'orientation sexuelle), recensent pourtant de nombreuses situations témoignant d'un profond mal-être vécu par des jeunes LGBT ou en questionnement sur leur orientation sexuelle. Le rapport annuel de l'association SOS 
homophobie, qui ne prétend pas être exhaustif et ne rapporte qu'une infime part des actes homophobes, évoque nombre de cas dramatiques, parmi lesquels des situations de rejet et d'exclusion des jeunes par leurs familles. En dépit du tableau assez sombre que ces associations permettent d'appréhender un peu, les travaux universitaires et les actions de prévention de l'homophobie demeurent rares.

C'est dans ce contexte que nous avons souhaité mener une enquête portant sur les liens entre l'homophobie en milieu scolaire et le suicide des jeunes. Parallèlement à ce travail de recherche, j'interviens également sur le terrain, dans le cadre d'une association qui met en place des actions pour prévenir l'homophobie et ses conséquences. Ces deux axes de travail m'apparaissent tout à fait complémentaires. C'est pourquoi je présenterai les interventions de l'association Contact, qui s'inscrivent dans le champ de l'éducation et de la formation, après avoir évoqué le contexte, le déroulement et les principaux résultats de la recherche que nous avons menée auprès de 901 jeunes à Toulouse.

\section{Présentation de l'enQuête et des PRINCIPAUX RÉsultats}

Cette enquête a été conduite dans le cadre d'une thèse de doctorat sous la direction de Chantal Zaouche et Sylvie Bourdet Loubère, respectivement professeure et maître de conférences à I'Université de Toulouse Le Mirail. Le professeur Jean-Philippe Raynaud, chef du service de psychiatrie de l'enfant et de l'adolescent du Centre hospitalier universitaire de Toulouse, a aussi contribué à la réalisation de cette recherche. Miguel Urdanoz, professeur d'économie l'École supérieure de commerce de Toulouse, est intervenu au moment de l'analyse des résultats quantitatifs. À notre connaissance, il s'agit de la première enquête universitaire française portant spécifiquement sur la problématique du suicide des jeunes en lien avec l'homophobie en milieu scolaire.

Pourtant, depuis une quinzaine d'années, les recherches sur les liens entre orientation sexuelle et conduites suicidaires se sont multipliées, essentiellement en Amérique du Nord. Si les toutes premières enquêtes ont été contestées en raison de biais méthodologiques, d'autres, plus solides, leur ont succédé et ont mis en évidence un phénomène de sursuicidalité chez les jeunes homosexuel-le-s ou bisexuel-le-s, particulièrement chez les sujets de sexe masculin (Beck et al., 2010).

Il va de soi que, dans le suicide d'un(e) jeune homosexuel-le, comme pour n'importe quelle autre personne, divers facteurs peuvent être impliqués sans aucun lien avec leur orientation sexuelle. Néanmoins, les auteur-e-s qui ont recensé les études sur ce thème soulignent qu'elles rapportent avec récurrence un risque suicidaire significativement plus élevé dans cette population, ce qui laisse supposer que cette population serait plus exposée que d'autres à des facteurs spécifiques (Verdier et Firdion 2003a; Beck et al., 2010). Selon ces auteur-e-s, l'hostilité à l'égard des personnes homosexuelles, qui prend des formes différentes et que l'on désigne le plus souvent par le terme d'homophobie, pourrait être à l'origine du phénomène observé. Cette hypothèse apparaît comme la plus souvent avancée et la plus étayée à ce jour. 
Le terme homophobie recouvre différentes notions, telles que des manifestations de haine, des propos, des actes de discrimination (Tin, 2003). D'après notre revue de la littérature, ce concept n'a jamais été opérationnalisé en France dans le cadre d'une recherche quantitative publiée. Par ailleurs, plusieurs auteurs s'interrogent au sujet de la définition de l'homophobie et la complètent, en soulignant, par exemple, que ce concept peut correspondre à un rejet de la non-conformité de genre chez les garçons (Welzer-Lang, 2002; Verdier et Firdion, 2003a). Ainsi, par exemple, un garçon perçu comme insuffisamment conforme aux critères de virilité déterminés par la société peut être victime d'homophobie. Dans cette perspective, les homosexuel-le-s ne seraient pas les seules personnes à présenter le risque d'être exposées à l'homophobie et il nous apparaît pertinent de tester les effets de cette dernière sur les jeunes adultes, quelle que soit leur orientation sexuelle.

Les auteurs qui ont conduit des recherches qualitatives portant sur le suicide des jeunes homo/bisexuel-le-s rapportent des témoignages qui interpellent, en révélant des parcours de vie marqués par la confrontation à de nombreux problèmes liés à l'homophobie, et cela particulièrement à l'adolescence (Dorais, 2001; Héfez, 2003; Verdier et Firdion, 2003a). II semble que ce vécu douloureux prenne souvent une dimension traumatique et se traduise encore plusieurs années après, dans les vicissitudes de la vie familiale, sociale, et professionnelle, comme l'analyse notamment la psychothérapeute Castañeda (1999). Ainsi, l'inscription durable du stigmate de l'homophobie pourrait contribuer à expliquer des conduites à risque et des comportements suicidaires survenant bien après les événements les plus difficiles auxquels le sujet a eu à faire face au cours de sa vie. Aussi, nous avons souhaité conduire notre recherche au moment charnière de la sortie de l'adolescence et du passage à l'âge adulte, dans la tranche d'âge des 18-24 ans, au travers d'une évaluation diachronique, à la fois rétrospective et actuelle, selon la nature des variables en jeu.

Après avoir analysé la littérature portant sur cette question, on retiendra que l'homophobie a un retentissement au niveau psychologique. Elle peut conduire à une profonde atteinte de l'estime de soi (Verdier et Firdion, 2003a) et se présenter sous forme d'homophobie intériorisée, résultant du conflit entre la prise de conscience d'une attirance homo/bisexuelle et les représentations négatives de l'homosexualité, acquises et intégrées par le sujet depuis le plus jeune âge (Chauvin, 2003; Tin, 2003). L'homophobie intériorisée se caractérise notamment par un ressenti très négatif sur sa propre homosexualité, associé à des sentiments de honte, de culpabilité, de non-conformité à l'idéal parental...

Dès lors, l'acte autoagressif, voire autodestructeur, pourrait correspondre à un comportement de fuite, à la volonté d'attaquer, de tuer, non pas soi, mais une partie de soi, ou l'autre en soi. Il se peut aussi que le passage à l'acte s'inscrive dans un contexte d'impulsivité en lien avec des événements déclenchants ou précipitants (Firdion, 2001). Des actes d'intimidation - phénomène mieux connu sous le nom de bullying ou d'intimidation - pourraient constituer l'un de ces événements, particulièrement lorsque ces actes comportent un caractère homophobe. Certains témoignages (Dorais, 2001) et des travaux ayant pour objet de recherche cette forme d'intimidation, de brimades, qui s'exprime notamment en milieu scolaire (Chamberland, 2011), nous le laissent supposer. 
Un article de Breton, paru dans Le Clinicien, en 2004, souligne que l'intimidation cible des jeunes vulnérables et peut conduire à des suicides. Il reprend la définition que donne Olweus en 1994 : « Un jeune est victime d'intimidation lorsqu'il est exposé de façon répétée et continue à des actions négatives de la part d'une ou de plusieurs personnes (Olweus, 1994, in Breton, 2004, 105). Il peut s'agir d'actions négatives directes: verbales ou physiques, ou indirectes, telles que l'exclusion sociale, les calomnies, les fausses rumeurs... (Breton, 2004).

En France, dans le rapport sur la violence scolaire remis l'an dernier au ministère de l'Éducation nationale, le concept de school bullying est plus simplement défini, de la manière suivante : « harcèlement et maltraitance entre pairs à l'école » (Bauer et al., 2010, 9).

S'appuyant sur la littérature scientifique, Bauer et al. (2010) rapportent des conséquences en termes de santé physique et mentale, évoquant notamment l'altération de fonctions cognitives telles la mémoire et la concentration, des troubles du métabolisme ou du système immunitaire, la baisse de l'estime de soi, les comportements suicidaires, le stress et la dépression.

Bien que l'on dispose à ce jour de peu de données sur ce sujet, il semble qu'il existe des liens étroits entre intimidation en milieu scolaire et homophobie. En effet, on peut penser que l'homophobie, très présente chez les garçons adolescents, participe au climat d'intimidation et accroît le niveau de violence en milieu scolaire.

Les résultats issus d'une recherche menée au Québec par Chamberland et al. (2011), auprès de 2747 élèves dans 30 écoles du deuxième cycle secondaire tendent à confirmer cette hypothèse. Dans un échantillon composé de $52,6 \%$ de filles et de $47,4 \%$ de garçons, il a notamment été demandé aux répondant-e-s à quelle fréquence, et pour quelles raisons, des élèves se faisaient taquiner méchamment, «écœurer », intimider, insulter, ou harceler. L'orientation sexuelle et la non-conformité de genre figurent parmi les trois critères les plus souvent cités par les élèves. Huit élèves sur dix rapportent que des élèves de leur école sont victimisés «parce qu'ils sont ou qu'on pense qu'ils sont gais, lesbiennes ou bisexuel-le-s ». Obtenus dans un échantillon où les élèves se sont pourtant déclarés très majoritairement hétérosexuel-le-s (92\%), ces résultats montrent l'importance que prend l'homophobie dans le phénomène plus global du bullying en milieu scolaire. Comme le souligne l'auteure, « c'est aussi à travers leur écho dans l'école que les incidents de violence homophobe déteignent sur le climat scolaire » (Chamberland et al., 2011, 1).

En France, jusqu'ici, l'homophobie en milieu scolaire n'a pas fait l'objet de recherches spécifiques, bien que, ces dernières années, elle semble devenue une préoccupation croissante de l'institution. Elle apparaît tout d'abord dans le cadre des documents relatifs à l'Éducation à la vie affective et sexuelle. Ainsi, le ministère de l'Éducation nationale y fait référence dans des circulaires, depuis 2001.

En l'absence d'enquête officielle, plusieurs indicateurs laissent entrevoir l'ampleur du phénomène et son ancrage en milieu scolaire. Par exemple, le rapport sur les discriminations en milieu scolaire mentionne que, « de manière générale, il ressort des auditions que les propos homophobes se sont banalisés ces dernières années » (Rebeyrol et al., 2010, 21). II souligne 
que les actes homophobes sont difficiles à recenser, notamment en raison des réticences des victimes à les déclarer. En 2011, un rapport sur le harcèlement en milieu scolaire a été remis par Debarbieux au ministre de l'Éducation nationale. Celui-ci mentionne que « sur le plan de la différence, seules les recherches sur les adolescents et adolescentes homosexuels ou considérés comme tels par leurs pairs ont montré un net lien avec l'intimidation, dans une homophobie pouvant être brutale ou verbale »(Debarbieux, 2011, 17). Ayral (2009) est l'auteure d'une thèse en sciences de l'éducation, intitulée « La fabrique des garçons, sanctions et genre au collège ». Elle a mené sa recherche dans cinq collèges de Bordeaux et de sa région, aux caractéristiques différentes. Elle dresse le constat suivant: les violences homophobes existent dans tous les établissements dans lesquels elle a enquêté, et cela sous des formes diverses (insultes, coups, attouchements, intimidations, etc.).

Ainsi, ces dernières années, plusieurs auteur-e-s ont fait part de leurs préoccupations au sujet des problèmes posés par l'homophobie auprès des jeunes scolarisé-e-s, notamment quand elle prend le prend le visage de l'intimidation (Ayral, 2009; Lert, 2009; Rebeyrol et al., 2010). Dès lors, dans le contexte français où le suicide des jeunes et les violences dans les établissements scolaires constituent des sujets majeurs de préoccupation, il paraît essentiel d'interroger le lien entre intimidation homophobe et suicide des jeunes. Nous avons donc choisi de centrer notre recherche sur ce thème.

\section{Recueil des données}

L'enquête s'est déroulée à Toulouse, une des plus grandes villes universitaires du pays, qui accueille plus de 100000 étudiant-e-s. Nous avons souhaité disposer d'un échantillon suffisamment important, soit environ un millier de participant-e-s. Pour le recrutement des sujets, nous avons choisi une structure recevant un large public de jeunes et qui pouvait mettre à notre disposition de bonnes conditions matérielles et un certain confort pour la passation des questionnaires. Le Centre Régional d'Information Jeunesse Midi-Pyrénées (CRIJ) répond à ces critères. Situé au centre-ville de Toulouse, c'est un lieu très fréquenté, avec plus de 400000 entrées par an. Les jeunes viennent y chercher des informations, consulter des offres d'emploi, de logement, de stages.. Le CRIJ propose également différents services tels qu'un relais Caisse d'allocations familiales / Locapass, une billetterie voyages, ou encore plusieurs postes d'accès à internet. Lors de leur passage dans les locaux du CRIJ, il a été proposé à tout-e-s les jeunes âgé-e-s de 18 à 24 ans de compléter un questionnaire autoadministré sur ordinateur. Environ un tiers d'entre eux a accepté de participer.

Trois postes informatiques ont été mis en place pour les besoins de cette recherche. La disposition et le choix du matériel permettaient d'assurer une confidentialité des réponses. De plus, la passation du questionnaire, totalement anonyme, donnait lieu à un enregistrement automatique des réponses sous forme codée dans un tableur Excel. Ce procédé, mis en œuvre par un informaticien, a permis d'éviter certains biais de confidentialité inhérents aux entretiens en face à face et permet également un codage des données plus rigoureux qu'il ne l'aurait été avec un questionnaire autoadministré complété à la main. 
La passation du questionnaire avait lieu en ma présence dans les locaux, ce qui permettait aux participant-e-s de me poser d'éventuelles questions sur le déroulement de l'enquête ou de disposer d'une écoute ou d'une réorientation vers des structures d'accueil, au cas où ils/elles exprimaient des questionnements ou un malaise liés aux questions posées.

Pour le recueil des données, nous avons eu recours à divers outils, essentiellement des questionnaires nord-américains, car, le plus souvent, aucun outil validé n'existait en France pour l'évaluation des variables étudiées. Nous avons également rédigé quelques items, en nous inspirant d'outils existants.

Le questionnaire, ainsi composé de 149 items, intégrait notamment les échelles Adolescent Risk Inventory (ARI, Lescano et al., 2007), Suicidal Behaviors Questionnaire - Revised (SBQ-r, Linehan, 1989), et Internalized Homophobia Scale (Martin et Dean, 1988).

Pour évaluer l'orientation sexuelle, nous avons principalement utilisé une échelle inspirée de l'échelle de Kinsey (1948), qui permet à l'origine de distinguer sept niveaux d'orientation sexuelle sur la base du degré d'attirance pour l'un ou l'autre des deux sexes, allant de « exclusivement hétérosexuel » jusqu'à « exclusivement homosexuel ». Nous l'avons réduite à 5 niveaux, par souci de simplification et de lisibilité des résultats et nous avons ajouté la possibilité de répondre « Je ne sais pas ».

Pour évaluer l'intimidation homophobe en milieu scolaire, nous nous sommes inspiré d'un outil réalisé par l'Association canadienne de santé publique (ACSP) : la trousse d'évaluation de l'intimidation, du harcèlement et des relations entre enfants du même âge en milieu scolaire (Totten, Quigley et Morgan, 2004). Parmi les exemples de harcèlement sexuel, cités par Totten et ses collègues (2004), figurent notamment les insultes au sujet de l'orientation sexuelle. Pour notre étude, nous nous sommes inspirés de la formulation de certains items du questionnaire de l'ACSP destiné aux élèves de la $8^{\mathrm{e}}$ à la $12^{\mathrm{e}}$ année. Nous avons uniquement retenu des questions se rapportant à l'intimidation en milieu scolaire en général, ou à ses formes plus spécifiquement homophobes, en les reformulant. Par exemple, l'item évaluant l'intimidation verbale homophobe chez les garçons était formulé ainsi : «Au collège ou au lycée, est-ce qu'un ou plusieurs autres jeunes vous ont traité de "pédé", de "tapette", de "tafiole", de "sale gay", ou d'une autre injure homophobe ? » Chacune des questions était assortie de quatre modalités de réponse: « Jamais », « Parfois », « Assez souvent », « Très souvent », que nous avons choisi de regrouper ensuite deux par deux pour nos analyses de la manière suivante: 1) Jamais/parfois; 2) Souvent/très souvent, afin de prendre en compte les formes d'intimidation les plus fréquentes, répondant le mieux à la définition des phénomènes de bullying.

Les conduites suicidaires recouvrent un ensemble de comportements depuis l'idéation suicidaire jusqu'au meurtre accompli de soi. Dans le cadre de cette étude, nous nous sommes intéressé au comportement suicidaire défini en termes d'idées, de tentative de suicide et de risque suicidaire. C'est pourquoi nous avons fait le choix d'utiliser l'échelle Suicide Behaviors Questionnaire, dans sa version révisée (SBQ-R) (Linehan, 1989). Ce questionnaire très court permet en effet d'obtenir un spectre assez large d'informations relatives aux comportements suicidaires. II présente aussi l'avantage de prendre en compte la temporalité, à travers 
l'évaluation de la récence des idéations et de la probabilité de se suicider un jour. Le premier item, quant à lui, permet de distinguer assez précisément différents niveaux d'idéations et de comportements suicidaires.

D'après certaines enquêtes sur l'intimidation en milieu scolaire, celle-ci peut avoir des effets sur la santé mentale persistant plusieurs années après l'épisode d'intimidation. Notre étude évaluant l'intimidation homophobe en milieu scolaire de façon rétrospective, nous avons souhaité déterminer si cette forme d'intimidation a un effet sur les tentatives de suicide au cours de la vie entière et si elle présente un effet quant au risque suicidaire actuel. Pour évaluer ce risque, nous nous sommes centré spécifiquement sur les participant-e-s rapportant des idéations suicidaires fréquentes au cours des douze derniers mois et une forte probabilité autoévaluée de se suicider un jour, afin de former une variable dichotomique « risque suicidaire actuel ».

Tout comme l'intimidation homophobe en milieu scolaire, l'homophobie intériorisée a été identifiée par certaines recherches comme un des facteurs du risque suicidaire chez les jeunes LGB. Aussi, nous avons souhaité tester si l'homophobie intériorisée présente un effet sur le risque suicidaire et, si tel est le cas, comparer cet effet avec celui de l'intimidation homophobe en milieu scolaire. Mais il convient de préciser que cette hypothèse n'a de sens que parce que nous l'avons testée auprès d'un échantillon restreint aux jeunes attiré-e-s par le même sexe, car l'homophobie intériorisée est une forme d'homophobie qui concerne uniquement cette population. Ainsi, lors de sa passation, le questionnaire informatisé autoadministré renvoyait automatiquement les participant-e-s ayant déclaré ressentir une attirance sexuelle pour le même sexe vers les questions de l'échelle d'homophobie intériorisée, tandis que les autres répondant-e-s passaient directement aux questions suivantes. De plus, l'homophobie intériorisée ne pouvant être évaluée rétrospectivement, nous avons testé son effet uniquement quant au risque suicidaire actuel, et non pas quant aux comportements suicidaires au cours de la vie.

\section{Principaux résultats}

L'analyse des résultats a porté sur 901 questionnaires, complétés par 475 filles et 426 garçons agé-e-s de 18 à 24 ans (âge moyen : 20,8 ans). La majorité d'entre eux/elles sont étudiant-e-s. C'est le cas de $77,9 \%$ des filles et de $63,2 \%$ des garçons. Concernant l'orientation sexuelle, on retiendra que les filles sont plus nombreuses que les garçons à déclarer ressentir une attirance sexuelle pour les deux sexes, tandis que les garçons sont plus nombreux à rapporter une attirance exclusive pour le même sexe comme le montrent les figures 1 et 2. Ce constat correspond à ceux des rares enquêtes sur la sexualité des jeunes en France (Lhomond, 1997). 
Figure

Orientation sexuelle chez les filles

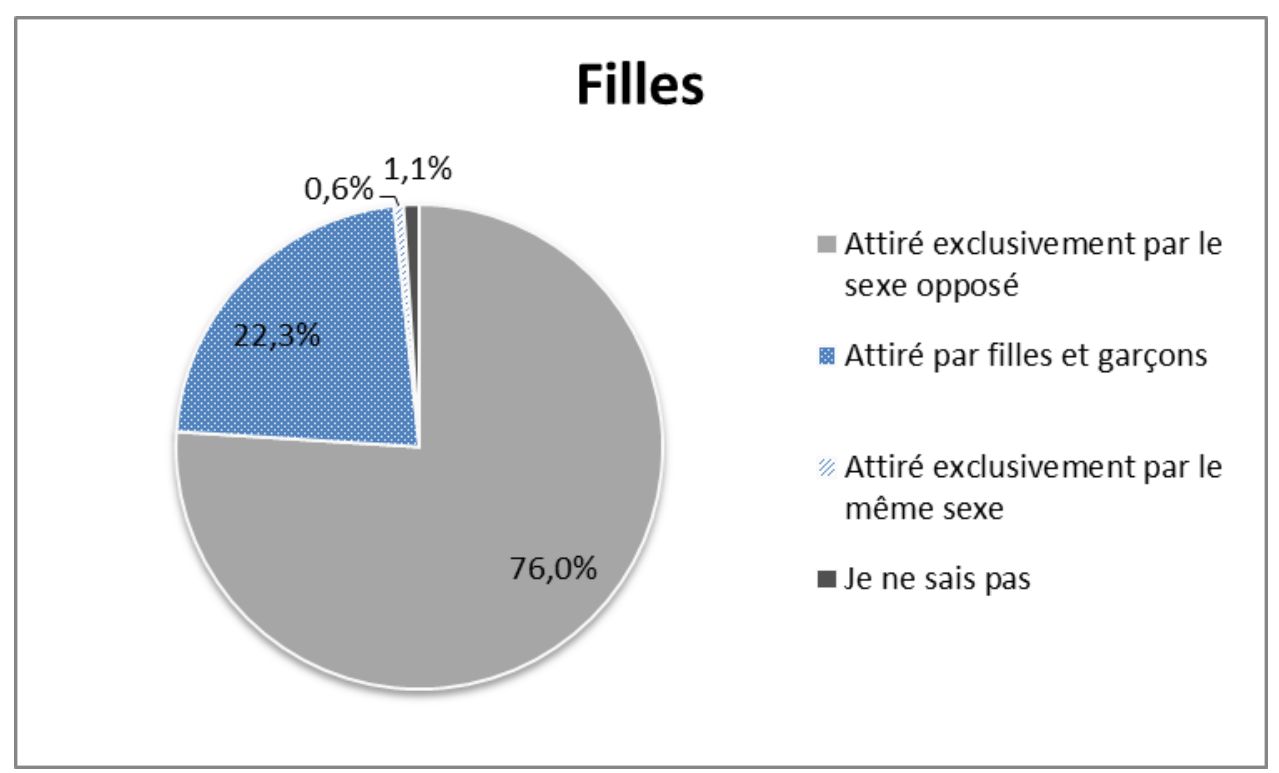

Figure 1

Orientation sexuelle chez les garçons

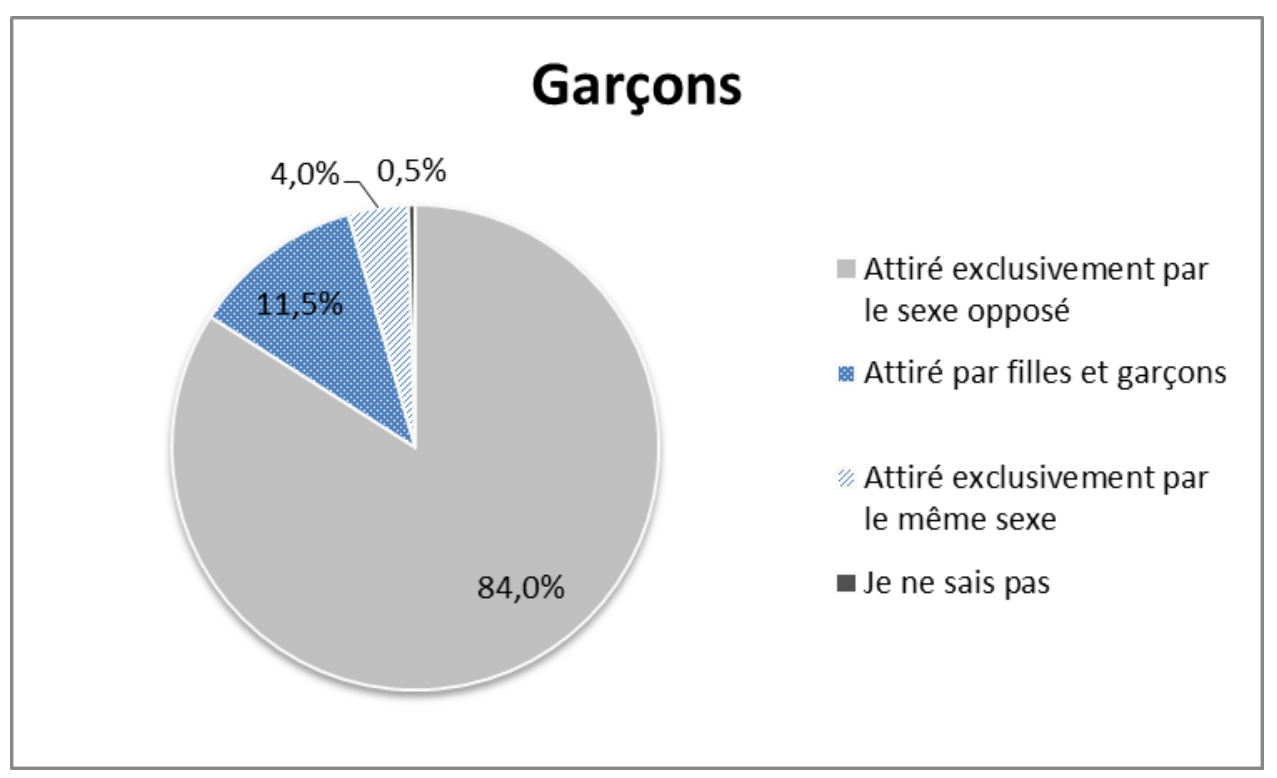

Afin de simplifier l'analyse des résultats et de prendre en compte des groupes d'effectifs plus conséquents, nous avons regroupé les participant-e-s qui déclarent ressentir une Attirance Sexuelle pour le Même Sexe ou être en Questionnement (groupe ASMSQ) que nous avons comparé au groupe des participant-e-s Attiré-e-s Sexuellement Exclusivement par le Sexe Opposé (ASESO). 
Cette comparaison nous a permis de confirmer, dans le contexte français, les tendances observées dans les grandes enquêtes menées en Amérique du Nord concernant le phénomène de sursuicidabilité des jeunes LGB. À partir des scores relevés à l'échelle des comportements suicidaires (SBQ-R), nous avons constaté que les idéations et tentatives de suicide étaient globalement plus présentes et plus graves chez les participant-e-s ASMSQ comme montre la figure 3. Différents résultats reflètent cette tendance. Ainsi, tandis que plus de la moitié des garçons et des filles ASESO n'ont jamais pensé au suicide, c'est le cas d'une minorité des filles ASMSQ $(28,9 \%)$ et de seulement un tiers des garçons ASMSQ.

Figure 2

Valeurs moyennes score total à l'échelle des comportements suicidaires

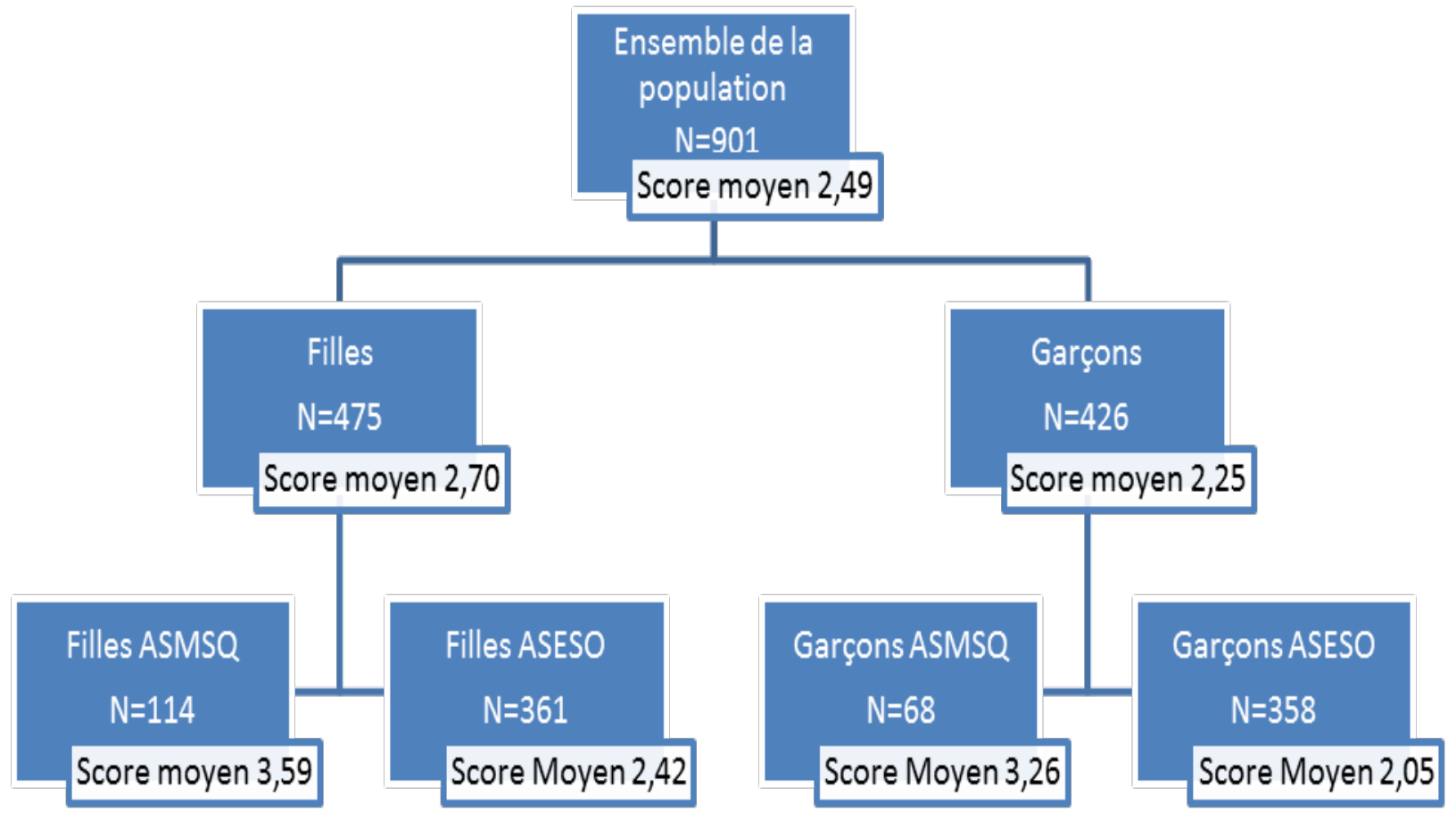


D'autre part, dans notre échantillon, les garçons sont significativement plus nombreux que les filles à rapporter avoir été victimes ou auteurs d'actes d'intimidation à caractère homophobe. Par exemple, alors que 25,7\% des garçons rapportent s'être déjà fait traiter de « pédé », de «tapette », de «tafiole », de « sale gay ou » d'une autre injure homophobe, seulement 5,5\% des filles rapportent avoir été victimes d'injures à caractère lesbophobe.

Afin de prendre en compte la notion de répétition des actes d'intimidation qui caractérise le phénomène de bullying, nous avons conduit les analyses en comparant les jeunes rapportant avoir subi les actes d'intimidation de manière fréquente (modalités de réponse « souvent » ou « très souvent ») avec ceux/celles rapportant « jamais », ou « parfois » en avoir été victime. La différence est alors encore plus importante concernant l'intimidation verbale homophobe/lesbophobe fréquente qui est huit fois plus répandue parmi les garçons que parmi les filles $(6,3 \%$ vs 0,8$)$.

Le recours à des analyses statistiques multivariées nous a permis de mettre en évidence que l'impact de l'intimidation verbale homophobe, fréquente en milieu scolaire, est plus fort chez les garçons, au point que l'effet de l'orientation sexuelle pour le même sexe sur les comportements suicidaires des jeunes hommes n'apparaît plus significatif dès lors qu'on introduit les variables «Intimidation verbale homophobe » et « Intimidation basée sur la nonconformité de genre ». Alors qu'elle ne représente pourtant qu'une des formes d'homophobie auxquelles les jeunes sont exposé-e-s à l'adolescence, l'intimidation verbale homophobe apparaît de façon tout à fait significative comme un facteur de risque suicidaire chez les jeunes hommes. Par ailleurs, ces variables « Intimidation verbale homophobe » et « Intimidation basée sur la non-conformité de genre » s'avèrent dans notre étude les seuls prédicteurs significatifs du risque suicidaire chez les garçons ASMSQ, tandis que chez les filles ASMSQ, il s'agit de la lesbophobie intériorisée. Ce dernier résultat est fort intéressant pour la compréhension des processus en jeu selon le sexe. Une des hypothèses qui permettrait de l'expliquer est que les filles et les garçons ne sont pas exposé-e-s de la même manière aux différentes formes d'homophobie à l'œuvre, du fait de l'interaction entre l'homophobie et le sexisme. Dans cette perspective, tandis que les garçons sont plus directement exposés aux actes d'intimidation homophobes, les filles souffriraient plutôt de l'homophobie sous sa forme intériorisée, moins visible. Ce dernier résultat va néanmoins devoir être confirmé par des recherches ultérieures puisqu'il se base sur un effectif réduit au sous-échantillon des participant-e-s ASMSQ.

Les autres analyses, en revanche, mesurent l'impact de l'intimidation homophobe en milieu scolaire sur les comportements suicidaires auprès de l'ensemble des participant-e-s, quelle que soit leur orientation sexuelle. C'est là, nous semble-t-il, l'un des apports majeurs de notre recherche, qui se place dans une perspective où l'homophobie ne concerne pas seulement les adolescents homo/bisexuels-le-s, mais qui considère qu'elle a un retentissement sur l'ensemble des jeunes scolarisé-e-s, comme le soulignent des recherches très récentes (Chamberland, 2011; Walton, 2010). En s'inscrivant dans ce paradigme, notre étude fait figure de pionnière en France où les données relatives au lien entre intimidation homophobe et suicide des jeunes font cruellement défaut. 
La richesse des données obtenues, du fait des choix méthodologiques qui ont présidé à notre démarche, pourra donner lieu à des analyses ultérieures, dans un contexte où l'homophobie constitue un vaste champ de recherche, presque inexploré. Elles pourront également être complétées par d'autres travaux. En effet, si l'intimidation verbale homophobe en milieu scolaire constitue, à elle seule, un facteur de risque suicidaire, l'homophobie a aussi d'autres conséquences au niveau social, qui peuvent à leur tour avoir un retentissement sur la santé mentale, l'estime de soi et sur les comportements autoagressifs des jeunes LGBT. Elle se caractérise notamment souvent par un manque de soutien familial. Cette dimension mériterait d'être explorée également dans le cadre d'études quantitatives portant sur un grand nombre de participant-e-s. Des travaux de recherche nous amènent à formuler l'hypothèse que l'anticipation d'un éventuel rejet par les parents peut s'avérer très fragilisant (Delor, 1999) et pourrait expliquer que la période pré-coming-out ait été identifiée comme particulièrement à risque (Hershberger et D'Augelli, 1995; Delor, 1999; Beck et al., 2010). II semble aussi qu'un fort niveau de soutien familial pourrait réduire l'impact de l'intimidation si celle-ci a une ampleur limitée (Hershberger et D'Augelli, 1995; Einsenberg et Resnick, 2006). Au contraire, lorsqu'il ou elle ne peut bénéficier du soutien de son entourage, l'adolescent-e homo/bisexuel-le est aussi particulièrement exposé-e au phénomène d'intimidation comprenant notamment les injures (Eribon, 1999; Hefez, 2003; Ryan et Frappier, 1994) et les agressions physiques (Hershberger et D'Augelli, 1995; D'Augelli et Grossman, 2001; Warner et al., 2004). Le manque de soutien des professionnels est aussi souligné (Lhomond, 1997; Castañeda, 1999), et cela peut s'avérer particulièrement problématique, notamment en milieu scolaire. Si l'on peut regretter l'absence de recherches universitaires de grande ampleur portant sur ces problématiques dans le contexte français, il convient néanmoins de souligner que plusieurs associations disposent de données issues des nombreux témoignages qu'elles ont pu collecter et des recensements qu'elles ont pu effectuer à partir des cas qui leur sont rapportés. Par ailleurs, ces associations mènent un important travail de terrain afin d'apporter une aide aux jeunes ou aux familles en difficulté. Si certaines structures, telles que SOS homophobie, recensent et luttent contre les actes homophobes dans leur ensemble, qu'il s'agisse d'agressions ou de discriminations, d'autres associations sont spécialisées dans le domaine de l'homophobie s'exprimant au niveau familial. C'est notamment le cas du Refuge, qui propose des hébergements et une prise en charge des jeunes qui ont été exclus du foyer familial quand leurs parents ont appris leur orientation sexuelle. L'association Contact, quant à elle, s'est créée dans les années 1990 afin de favoriser une compréhension mutuelle entre les jeunes homos ou bisexuel-le-s et leurs familles.

\section{Remerciements}

Nous adressons un grand remerciement à l'ensemble des partenaires qui nous ont apporté leur soutien dans le cadre de ce travail de recherche, notamment à l'équipe du Centre Régional d'Informations Jeunesse (CRIJ) de Midi-Pyrénées et à la Fondation Wyeth pour la santé de l'Enfant et de l'Adolescent. 


\section{EXEMPLES D'ACTIONS DE PRÉVENTION : L'ASSOCIATION CONTACT}

L'association Contact a pour originalité de rassembler des lesbiennes, des gays, des bisexuelle-s, mais aussi des parents, des grands-parents, des frères et sœurs, des proches et des amie-s de personnes homosexuelles ou bisexuelles. J'interviens dans le cadre de cette association familiale et inter-générationnelle depuis 2003. Elle a pour but d'aider les parents et familles à accepter l'homosexualité d'un proche, d'aider les personnes homosexuelles ou bisexuelles à mieux accepter leur orientation sexuelle, et de lutter contre l'homophobie.

Pour y parvenir, Contact met en place des actions destinées à répondre aux attentes de trois types de publics : les parents, les jeunes, et les professionnel-le-s qui interviennent auprès des jeunes et/ou des familles. L'association propose notamment une ligne d'écoute anonyme, des permanences d'accueil, des entretiens avec des bénévoles formés à l'écoute ou avec un psychologue, des réunions ouvertes sur le modèle des groupes de paroles, des interventions en milieu scolaire ou dans les structures d'éducation populaire, des brochures et des supports pédagogiques, des journées de formations et de sensibilisation des professionnel-le-s, des conférences-débat et des colloques. Le travail que cette association mène afin de prévenir les conséquences psychologiques et sociales de l'homophobie est largement reconnu et elle bénéficie de l'agrément « Jeunesse et Éducation Populaire » depuis 1999 et de l'agrément du ministère de l'Éducation nationale depuis 2008.

Depuis quelques années, pour faire face à son développement, Contact fonctionne sous forme d'un réseau comprenant une vingtaine d'associations réparties sur le territoire français. J'interviens dans cette association au niveau national, en tant que formateur des bénévoles intervenant en milieu scolaire, et au niveau local, en tant que coordinateur des actions éducatives, sur le territoire de la région Midi-Pyrénées.

Les actions de l'association Contact s'inscrivent pleinement dans un objectif de prévention du suicide des jeunes. En effet, comme nous l'avons évoqué dans la première partie de cet article, les recherches, qu'elles soient internationales ou locales, mettent en évidence le lien entre le suicide des jeunes et l'homophobie ou les difficultés d'acceptation de l'orientation sexuelle. Or les hypothèses formulées par les chercheurs pour expliquer ce lien font référence à plusieurs types de problèmes rencontrés à différents niveaux par les jeunes qui prennent conscience de leur attirance pour les personnes de même sexe.

Au niveau familial, la découverte de l'homosexualité par les parents peut entrainer un éventuel rejet, des situations de ruptures familiales, des problèmes de communication, ou un manque de soutien (Hershberger et D'Augelli, 1995; Delor, 1999; Beck et al., 2010). Il convient de souligner qu'avant de révéler leur homosexualité à des proches, les jeunes anticipent souvent ce risque de rejet. Par crainte d'être mis à la porte ou même, simplement, de décevoir leurs parents, ils/elles choisissent alors de ne pas en parler, ce qui les amène le plus souvent à mentir et complique encore leur vie quotidienne. Cette situation engendre du stress et de l'anxiété, des émotions susceptibles de contribuer à les fragiliser. 
Au niveau scolaire, l'exposition à l'homophobie peut expliquer certains comportements suicidaires des adolescent-e-s et jeunes adultes, comme le confirment les résultats de l'enquête que nous avons menée à Toulouse. On soulignera notamment que l'intimidation verbale à caractère homophobe, fréquente et souvent banalisée, augmente significativement le risque suicidaire chez les adolescents et jeunes adultes de sexe masculin.

Au niveau intra-psychique, on peut penser que l'homophobie intériorisée contribue également au passage à l'acte suicidaire en raison de l'atteinte à l'estime de soi qui caractérise cette période marquée par le conflit entre prise de conscience d'une attirance homosexuelle et la présence de préjugés homophobes intériorisés depuis l'enfance. De plus, les sentiments de honte et de culpabilité peuvent se doubler d'un certain isolement qui fragilise encore ces jeunes.

On pourra remarquer qu'à chacun de ces différents niveaux correspond un des objectifs de l'association Contact. En effet, celle-ci propose une écoute adaptée aux familles, mais aussi aux jeunes qui se découvrent homosexuel-le-s ou bisexuel-le-s, ainsi que des actions de prévention de l'homophobie en milieu scolaire.

Les actions de sensibilisation et de formation des professionnel-le-s, proposées par Contact dans notre région, complètent cette série d'interventions. Elles aident ces professionnel-le-s à mieux prendre en charge les situations de crise suicidaire liées à l'homophobie. Elles s'adressent aux professionnel-le-s intervenant dans le champ de l'éducation, de la famille, de la jeunesse, de la santé et de la prévention.

Les formations se composent d'un travail portant sur les représentations, d'apports théoriques (orientation sexuelle, homophobie intériorisée, hétérosexisme...), d'études de cas et d'analyses sur la base de supports vidéo et de situations rapportées par les professionnel-le-s. Chaque année, depuis trois ans, une centaine de professionnels en bénéficient, dans plusieurs villes de la région.

Les actions proposées aux familles visent, quant à elles, à restaurer le dialogue et à favoriser la compréhension mutuelle.

Quand elles découvrent l'homosexualité ou la bisexualité d'un proche, les familles ne sont pas préparées à cette éventualité et manquent de repères. Pour les aider, Contact met notamment en place des « réunions ouvertes à la parole » au cours desquelles les familles peuvent confronter leur expérience en rencontrant d'autres parents, d'autres proches, qui les aident à faire face à cette situation. Ces rencontres leur permettent aussi de dialoguer avec des lesbiennes, des gays et des bisexuel-le-s et de prendre connaissance de leurs parcours et de leurs préoccupations. Quant aux jeunes homosexuel-le-s, ils/elles peuvent ainsi découvrir comment d'autres parents, d'autres proches réagissent, afin d'envisager la meilleure façon d'en parler à leur famille et à leurs ami-e-s, s'ils/elles le souhaitent. À l'occasion de ces groupes de parole, Ils peuvent aussi mesurer les difficultés auxquelles ils/elles pourraient être confrontés-e$\mathrm{s}$ et mieux savoir comment les anticiper. 
Enfin, les actions menées en milieu scolaire par l'association Contact dans notre région s'inscrivent dans un objectif de prévention de l'intimidation à caractère homophobe. Elles se déroulent généralement sous forme d'interventions en groupe classe, d'une durée de deux heures environ. Ces interventions sont très interactives et animées par deux intervenant-e-s formées.

Après avoir défini les notions d'orientation sexuelle et d'homophobie, les élèves peuvent poser leurs questions par écrit. Ensuite un court-métrage est projeté et les intervenant-e-s leur proposent de réagir et de répondre aux questions posées par écrit, en favorisant leur réflexion. Après la projection d'un second court-métrage, les intervenant-e-s apportent souvent leurs témoignages afin d'illustrer le propos et de permettre aux élèves de mieux comprendre le vécu des personnes homosexuelles, bisexuelles, ou de leurs parents et proches.

Cette progression, depuis les définitions générales du thème jusqu'aux témoignages des intervenant-e-s, en passant par la projection de films courts, constitue une approche originale qui permet aux élèves d'évoluer dans leur perception de l'homosexualité et de prendre conscience de la gravité des conséquences de l'homophobie. Alors que les questions qu'ils/elles posent en début d'intervention témoignent parfois d'un niveau d'homophobie extrême, l'évaluation qu'ils/elles complètent en fin d'intervention nous encourage.

Par exemple, en 2011-2012, sur 3000 élèves sensibilisé-e-s :

L'intervention est jugée « intéressante » ou « très intéressante » par $93 \%$ des filles et $69 \%$ des garçons. On pourra remarquer, au passage, que ce résultat met en évidence une différence assez importante entre les réponses apportées par les filles et les garçons, et on pourra formuler l'hypothèse que le rôle joué par l'homophobie dans la construction du masculin à l'adolescence n'y est pas étranger.

Par ailleurs, $59 \%$ de filles et $45 \%$ de garçons estiment que l'intervention les a aidé-e-s personnellement et $95 \%$ de l'ensemble des élèves considèrent que l'intervention « peut aider d'autres personnes ». De plus, les commentaires écrits apportés par les élèves sur ces évaluations sont souvent très positifs et les établissements scolaires sont de plus en plus nombreux à faire appel à l'association. Ainsi, depuis six ans, Contact a mené plus de 500 interventions en région Midi-Pyrénées. Plus de 12000 élèves ont ainsi été sensibilisé-e-s, dans une soixantaine d'établissements de notre région. Néanmoins, il convient de préciser que notre région compte 550000 élèves scolarisé-e-s et que les autres associations qui interviennent en milieu scolaire sur le thème de l'homophobie sont très rares. En France, seules trois associations, dont Contact, disposent de l'agrément du ministère de l'Éducation nationale. Dans certaines régions, il n'existe même aucune structure de ce type.

Eu égard aux résultats obtenus qui sont très encourageants, on ne peut que regretter que le nombre d'élèves qui bénéficient de ce type d'interventions associatives demeure probablement très faible, une fois rapporté à la population globale des élèves scolarisé-e-s. Or, on peut penser, comme Chamberland, que l'absence d'interventions pour contrer ou prévenir la violence homophobe peut « laisser entendre que cette violence serait acceptable, sans gravité, voire légitime. » (Chamberland et al., 2011, 1). 
Les liens étroits entre suicide des jeunes et homophobie qui ont été mis en évidence par les recherches nous amènent à formuler le souhait que des initiatives soient prises et que des moyens bien plus importants soient accordés aux associations afin qu'elles puissent développer leurs actions. Le bien-être et la santé d'un grand nombre de jeunes en dépendent.

Jean-Michel Pugniere

Psychologue et docteur en psychologie, Toulouse - France 


\section{BIBLIOGRAPHIE}

Ayral, S. (2009). La fabrique des garçons. Sanctions et genre au collège. Thèse de doctorat en Sciences humaines et sociales, mention Sciences de l'Éducation, sous la direction du professeur E. Debarbieux, Université Segalen Bordeaux-2.

Bauer, A., C. Soullez, A. Anton, J.M. Bernard, E. Debarbieux, A. Forestier et E. Martin (2010). Rapport remis au ministre de l'Éducation nationale, Mission sur les violences en milieu scolaire, les sanctions et la place de la famille.

Beck, F., J.M. Firdion, S. Legleye et M.A. Schiltz (2010). Les minorités sexuelles face au risque suicidaire. Acquis des sciences sociales et perspectives. Dossiers Santé en action, INPES.

Breton, JJ. (2004). «Liaisons néfastes à l'école : victimes et intimidateurs ». Le Clinicien, mars 2004, p. 105-110.

Castañeda, M., (1999). Comprendre l'homosexualité. Des clés, des conseils pour les homosexuels, leurs familles, leurs thérapeute, Paris, Robert Laffont, coll. « Pocket ».

Chamberland, L., G. Émond, D. Julien, J. Otis et B. Ryan (2011). L'homophobie à l'école secondaire au Québec. Portrait de la situation, impacts et pistes de solution. Rapport de recherche.

Chauvin, S. (2003), dans Tin, L.G. (2003). Dictionnaire de l'homophobie. Paris, Presses universitaires de France, p. 222-226.

D'Augelli, A.R., S. Hershberger et N.W. Pilkington (2001). « Suicidability patterns and sexual orientation-related factors among lesbian, gay, and bisexual youths ». Suicide and Life Threatening Behaviour, $\mathrm{n}^{\circ} 31$, p. 250-264.

Debarbieux, E. (2011). Les états généraux de la sécurité à l'école. Comprendre, prévenir, agir. Fiches thématiques, contribution du Conseil scientifique. Ministère de l'Éducation nationale.

Delor, F. (1999). « Homosexualité et reconnaissance : le corps du mépris », dans C. Broqua, F. Lert et Y. Souteyrand (dir.) (2003). Homosexualités au temps du sida. Paris, Association nationale de recherches sur le sida et les hépatites virales (ANRS), coll. « Sciences sociales et sida », p. 273-292.

Dorais, M. (2001). Mort ou fif. La face cachée du suicide chez les garçons, Montréal, VLB éditeur.

Eisenberg, M., Resnick, M. (2006). « Suicidability among gay, lesbian and bisexual youth : the role of protective factors ». Journal of Adolescent Health, vol. $39, n^{\circ} 5$, p. 662-668

Eribon, D. (1999). Réflexions sur la question gay. Paris, Fayard.

Firdion, J.M. (2001) Le risque de suicide chez les jeunes à orientation sexuelle non conventionnelle. Groupe de travail sur le suicide des jeunes, HES. 
Hefez, S., «Adolescence et homophobie » (2003). dans C. Broqua, F. Lert et Y. Souteyrand (dir.), Homosexualités au temps du sida, Paris, ANRS, coll. "Sciences sociales et sida », p. 147-168.

Hershberger, S.L., et A.R. D'Augelli (1995). « The Impact of Victimization on the Mental Health and Suicidality of Lesbian, Gay and Bisexual Youths ». Developmental Psychology, APA, vol. $31, n^{0} 1$, p. 65-74.

Kinsey, A., W. Pomeroy et C. Martin (1948). Le comportement sexuel de l'homme, Paris, Éditions du Pavois.

Lert, F. (2003). «Traiter de l'homosexualité en milieu scolaire » dans C. Broqua, F. Lert et Y. Souteyrand (dir.), Homosexualités au temps du sida. Paris, ANRS, coll. "Sciences sociales et sida », p. 169-176.

Lert, F. (2010). «Se donne-t-on les moyens en France de lutter contre l'homophobie et de promouvoir le bien être des jeunes attirés par le même sexe ? ». Colloque Éducation et Homophobie, une forme de discrimination dans le système éducatif : États des lieux, actions et relais de l'action, Résumés et Abstracts, 36.

Lert, F. et G. Pialoux, (2009). Prévention et réduction des risques dans les groupes à haut risque vis-à-vis du VIH et des IST. Rapport de la mission RDRS.

Lescano, C., N. Beausoleil, L. Brown, D. D’Eramo, W. Hadley et A. Zimskind (2007). « A Brief Screening Measure of Adolescent Risk Behavior », Child Psychiatry and Human Development, $\mathrm{n}^{\circ}$ 37, p. 325-336.

Lhomond, B. (1997). «Attirances et pratiques homosexuelles » dans $\mathrm{H}$. Lagrange et B. Lhomond, L'entrée dans la sexualité. Le comportement des jeunes dans le contexte du sida. Paris, La Découverte, coll. « Recherches ».

Lhomond, B. et M.J. Saurel-Cubizolles (2003). «Orientation sexuelle, violences contre les femmes et santé : résultats de l'enquête nationale sur les violences envers les femmes en France », dans C. Broqua, F. Lert et Y. Souteyrand (dir.), Homosexualités au temps du sida. Paris, ANRS, coll. « Sciences sociales et sida », p. 107-130.

Martin, J.L.. et L. Dean (1987). Summary of measures : Mental health effects of AIDS on at-risk homosexual men. Division of socio-medical sciences, Columbia University, School of Public Health. Manuscrit non publié.

Pugnière, J.M. (2011) Orientation sexuelle et comportement suicidaire chez des jeunes adultes de sexe masculin. Étude de l'influence de l'homophobie et de l'homophobie intériorisée. Thèse de doctorat en psychologie, sous la direction de Sylvie Bourdet-Loubère Sylvie, maître de conférences, et de Chantal Zaouche, professeure. Université de Toulouse II Le Mirail.

Rebeyrol et al. (2010). Discriminations à l'École. Rapport relatif aux auditions sur les discriminations en milieu scolaire remis au ministre de l'Éducation nationale, le 22 septembre 2010. 
Ryan, B. et J.-Y. Frappier (1994). «Quand l'autre en soi grandit: les difficultés à vivre l'homosexualité à l'adolescence », dans D. Dorais, P. Dutey et D. Welzer-Lang, La peur de l'autre en soi. Du sexisme à l'homophobie, p. 238-251, Montréal, VLB éditeur, coll. « Des hommes et des femmes en changement ».

Tin, L.G. (2003). Dictionnaire de l'homophobie. Paris, Presses universitaires de France.

Totten, M. et P. Quigley (2005). Parental knowledge of child-reported bully-victim and sexual harassment problems in several Canadian schools : Implications for policy and program development. Canadian Public Health Association.

Totten, M., P. Quigley et M. Morgan (2004). Trousse d'évaluation de l'intimidation, du harcèlement et des relations entre enfants du même âge en milieu scolaire. Association canadienne de santé publique (ACSP).

Verdier, E. et J.M. Firdion (2003a). Homosexualités et suicide, Saint-Martin-de-Londres, H\&O Éditions.

Verdier, E. et J.M. Firdion (2003b). « Suicide et tentatives de suicide parmi les personnes à orientation homosexuelle » dans C. Broqua, F. Lert et Y. Souteyrand (dir.), Homosexualités au temps du sida, Paris, ANRS, coll. « Sciences sociales et sida », p. 157-168.

Walton, G. (2010). « Sécurisation des milieux d'apprentissage. Lutte contre l'intimidation à caractère homophobe dans les écoles. Faire la différence... », De la recherche à la pratique, $\mathrm{n}^{\circ}$ 30. Secrétariat de la littéracie et de la numératie, Ontario Association of Deans of Education.

Warner, J., E. McKeown, M. Griffin, K, Johnson, A. Ramsay, C. Cort et M. King (2004). « Rates and predictors of mental illness in gay men, lesbians and bisexual men and women », British Journal of Psychiatry, vol. 185, nº 6, p. 479-485

Welzer-Lang, D., (2002). « Virilité et virilisme dans les quartiers populaires en France », VEI Enjeux, $\mathrm{n}^{0} 128$, p. $10-32$ 